\title{
Potential of whole-genome sequencing-based pharmacogenetic profiling
}

\author{
Sylvan Manuel Caspar ${ }^{1,2}$ (D), Timo Schneider ${ }^{1}$, Patricia Stoll ${ }^{1}$, Janine Meienberg ${ }^{1}$ \& Gabor \\ Matyas*,1,3 (iD) \\ ${ }^{1}$ Center for Cardiovascular Genetics \& Gene Diagnostics, Foundation for People with Rare Diseases, Schlieren-Zurich 8952, \\ Switzerland \\ ${ }^{2}$ Department of Health Sciences \& Technology, Laboratory of Translational Nutrition Biology, ETH Zurich, \\ Schwerzenbach 8603, Switzerland \\ ${ }^{3}$ Zurich Center for Integrative Human Physiology, University of Zurich, Zurich 8057, Switzerland \\ *Author for correspondence: matyas@genetikzentrum.ch
}

Pharmacogenetics represents a major driver of precision medicine, promising individualized drug selection and dosing. Traditionally, pharmacogenetic profiling has been performed using targeted genotyping that focuses on common/known variants. Recently, whole-genome sequencing (WGS) is emerging as a more comprehensive short-read next-generation sequencing approach, enabling both gene diagnostics and pharmacogenetic profiling, including rare/novel variants, in a single assay. Using the example of the pharmacogene CYP2D6, we demonstrate the potential of WGS-based pharmacogenetic profiling as well as emphasize the limitations of short-read next-generation sequencing. In the near future, we envision a shift toward long-read sequencing as the predominant method for gene diagnostics and pharmacogenetic profiling, providing unprecedented data quality and improving patient care.

\section{Graphical abstract:}

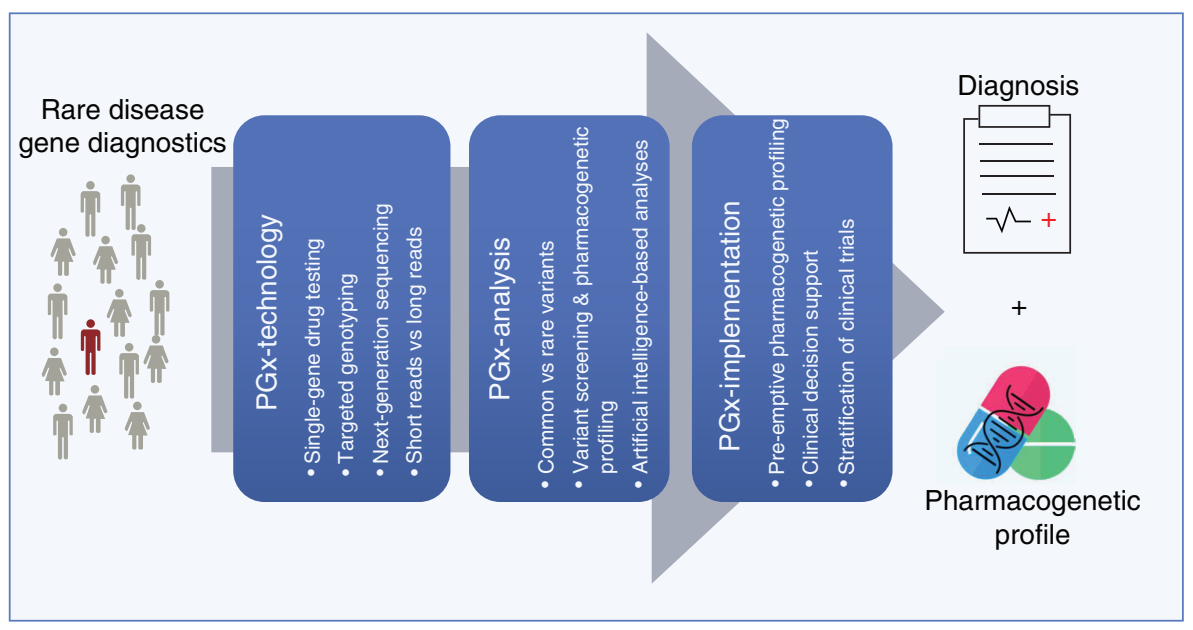

First draft submitted: 12 October 2020; Accepted for publication: 9 November 2020; Published online: 1 February 2021

Keywords: adverse drug reactions • CYP2D6 - long-read sequencing • next-generation sequencing - pharmacogenetics $\bullet$ precision medicine $\bullet$ pseudogenes $\bullet$ structural variation

\section{Current state of pharmacogenetics}

The term pharmacogenetics (PGx) first appeared in scientific literature in 1959 [1] and describes the field of genetics that investigates the effect of genetic variability on individual drug response [2]. PGx includes germline variants which influence the pharmacokinetics and pharmacodynamics of pharmaceuticals as well as somatic tumor-driver variants that influence the response to cancer drug therapies [3].

At present, the Pharmacogenomics Knowledgebase (PharmGKB) lists 68 clinically important pharmacogenes, $>100$ gene-drug pairs and $>700$ annotated drugs (pharmgkb.org, accessed October 2020), integrating information 
from various PGx resources, such as the Dutch Pharmacogenetics Working Group (DPWG, knmp.nl) and the Clinical Pharmacogenetics Implementation Consortium (CPIC, cpicpgx.org). Pharmacogenes may be classified according to their function into categories, such as encoding Phase I (e.g., CYP) and Phase II (e.g., UGTs) enzymes, transporters (e.g., $A B C B 1$ ), drug targets (e.g., VKORC1), oncogenes (e.g., $E G F R$ ) or involved in the immune system (e.g., $H L A-B$ ) [4]. Detected (functional) variants are primarily described using the star $\left(^{*}\right)$ allele nomenclature in most pharmacogenes [5]. In PGx, differences in enzyme activity lead to varying phenotypes, according to which individuals are classified as poor metabolizer (PM), intermediate metabolizer, normal metabolizer (NM) or ultrarapid metabolizer [2].

The importance of PGx may be highlighted by several facts. For instance, adverse drug reactions are a substantial socio-economic burden, being responsible for up to $\sim 12.8 \%$ of hospital admissions in the EU, while approximately $0.5 \%$ of adverse drug reactions are lethal [6]. Indeed, as almost every individual harbors at least one actionable sequence variant according to the DPWG or CPIC guidelines [7,8], PGx could be implemented for patient stratification in clinical care and clinical trials [7]. Nevertheless, the majority of drugs are currently prescribed without the integration of pharmacogenetic information.

Pre-emptive pharmacogenetic profiling offers better patient care, enabling the incorporation of PGx into clinical decision support (CDS). A plethora of approaches for pharmacogenetic profiling are available and recent technical advances in next-generation sequencing (NGS) enable the integration of PGx into clinical settings more than ever. Currently, however, the benefits of PGx from randomized controlled trials (RCTs) and meta-analyses are mostly limited to single drug-gene pairs and the genes/variants that should be tested for the optimal prediction of drug response are not standardized [4,9]. In addition, there is low inter-laboratory concordance of test results [9] and the abundance of options for the implementation of PGx can be overwhelming.

Here, we review the methods of PGx and explore the potential of pharmacogenetic profiling based on our wholegenome sequencing (WGS) pipeline [7], which enables the combination of comprehensive molecular diagnostics and pharmacogenetic profiling in a single assay. Moreover, using the example of the pharmacogene CYP2D6, we outline the challenges and opportunities of current pharmacogenetic profiling methods and discuss potential future directions.

\section{Genotyping of pharmacogenetic variants}

Traditional methods

After the first discovery of the biochemical basis of pharmacogenetic variation in the 1970s in the context of debrisoquine hydroxylation [10] and sparteine oxidation [11] by the enzyme later termed CYP2D6, the first pharmacogenetic test was made available in 1990 [12]. This test was developed to identify CYP2D6 PMs and was based on allele-specific PCR. Similarly, in the following years, more functional variants were identified in other pharmacogenes by means of PCR-based methods and Sanger sequencing. From the late 1990s, microarrays have enabled increased throughput and parallelized genotyping of common/known variants across several genes [4,13-16]. Meanwhile, a variety of molecular methods have been adopted for the research and diagnostic applications of PGx (Table 1).

In the absence of regulation and clear guidelines, the applied methods and interrogated genes/variants may vary among testing laboratories, leading to inconsistent results [9]. Typically, the tested variants are selected according to the indication or the drug class, such as antihypertensives, psychotropics or anticoagulants [4]. From an economic perspective and particularly considering possible re-use of results [24], pre-emptive testing of multiple variants is superior to a single gene-drug approach [9]. Due to their cost efficiency, scalability, short turnaround time and high clinical utility, PCR- and microarray-based targeted genotyping (hereafter collectively referred to as targeted genotyping) has frequently been applied for pharmacogenetic profiling. For instance, in the ubiquitous pharmacogenomics (U-PGx) program, which aims to provide evidence for the clinical utility of pre-emptive pharmacogenetic profiling, a PCR-based assay is being applied for pharmacogenetic profiling [25]. Modern microarrays may be limited to pharmacogenetic variants (e.g., AmpliChip CYP450 [Roche, Basel, BS, Switzerland], PharmacoScan [ThermoFisher, MA, USA]), or interrogate close to 1 million variants (e.g., Infinium Global Screening Assay 3.0 [Illumina, CA, USA], Genome-Wide Human SNP Array 6.0 [Affymetrix, CA, USA]) and may be leveraged for pharmacogenetic profiling of large-scale biobanks [8,26].

A major caveat of targeted pharmacogenetic profiling is the subset of interrogated variants. The selection of variants to be tested may be decisive for treatment outcome, as illustrated by the conflicting results of several studies aiming to assess the efficacy of PGx-guided compared with clinically guided dosing of the anticoagulant warfarin. 


\begin{tabular}{|c|c|c|c|}
\hline & Advantages & Disadvantages & Ref. \\
\hline Traditional methods & $\begin{array}{l}\text { - Low costs, short turnaround time, interpretation } \\
\text { of results }\end{array}$ & $\begin{array}{l}\text { - Limited to the subset of tested } \\
\text { variants/fragments }\end{array}$ & \\
\hline $\begin{array}{l}\text { PCR (e.g., Luminex xTAG CYP2D19/CYP2D6, LGC } \\
\text { SNPline) }\end{array}$ & $\begin{array}{l}\text { - High sensitivity } \\
\text { - Variety of available PCR-based methods (TaqMan, } \\
\text { long-range PCR, quantitative real-time PCR, digital } \\
\text { PCR) }\end{array}$ & - Low/limited throughput & [15] \\
\hline Sanger sequencing & $\begin{array}{l}\text { - Allows sequencing with single-base-pair } \\
\text { resolution } \\
\text { - Combination with long-range PCR for allele } \\
\text { specificity }\end{array}$ & $\begin{array}{l}\text { - Low/limited throughput } \\
\text { - Laborious }\end{array}$ & [15] \\
\hline $\begin{array}{l}\text { Multiplex ligation-dependent probe amplification } \\
\text { (MLPA) }\end{array}$ & - Semi-quantitative detection of CNVs & $\begin{array}{l}\text { - Low/limited throughput and limited to } \\
\text { probe-binding sites } \\
\text { - Laborious, sensitive to handling }\end{array}$ & [17] \\
\hline Mass spectrometry (e.g., MALDI-TOF) & $\begin{array}{l}\text { - High throughput (option for automation) } \\
\text { - High sensitivity }\end{array}$ & $\begin{array}{l}\text { - Expensive equipment, no US FDA-approved } \\
\text { platform }\end{array}$ & [18] \\
\hline $\begin{array}{l}\text { Microrrays (e.g., ThermoFisher PharmacoScan, } \\
\text { Affymetrix Genome-Wide Human SNP Array) }\end{array}$ & - High throughput (option for automation) & $\begin{array}{l}\text { - Complex structural variants remain } \\
\text { undetected (e.g., CYP2D6*13,*68) }\end{array}$ & \\
\hline $\begin{array}{l}\text { Short-read NGS (e.g., Illumina, lon Torrent, BGI } \\
\text { Genomics) }\end{array}$ & - Detection of rare/novel variants & - Turnaround time, interpretation of results & \\
\hline $\begin{array}{l}\text { Targeted sequencing (e.g., Ion AmpliSeq } \\
\text { Pharmacogenomics Research Panel, PGRNseq) }\end{array}$ & $\begin{array}{l}\text { - Cost efficient } \\
\text { - Existing PGx-specific analysis tools (for PGRNseq) }\end{array}$ & $\begin{array}{l}\text { - Separate assays for diagnostics and } \\
\text { pharmacogenetic profiling } \\
\text { - Enrichment laborious }\end{array}$ & [19] \\
\hline WES & $\begin{array}{l}\text { - Increased throughput } \\
\text { - Cost efficient (molecular diagnosis and } \\
\text { pharmacogenetic profiling in one assay) }\end{array}$ & $\begin{array}{l}\text { - Incomplete coverage of exome } \\
\text { - Non-exonic variants are not interrogated } \\
\text { (e.g., CYP2C19*17, CYP3A5*3, } \\
\text { UGT1A1*28/*37 and VKORC } 1 * 2 \text { ) }\end{array}$ & [7] \\
\hline WGS & $\begin{array}{l}\text { - Existing PGx-specific analysis tools } \\
\text { - Molecular diagnosis and pharmacogenetic } \\
\text { profiling in one assay }\end{array}$ & $\begin{array}{l}\text { - Read alignment and variant calling in } \\
\text { homologous/repetitive regions (e.g., CYP and } \\
\text { UGT genes) }\end{array}$ & [7] \\
\hline Long-read NGS (e.g., PacBio, ONT) & $\begin{array}{l}\text { - Phasing, variant detection in } \\
\text { homologous/repetitive regions }\end{array}$ & $\begin{array}{l}\text { - Raw-read error rate (ONT), costs, input } \\
\text { material (PacBio) }\end{array}$ & \\
\hline Targeted sequencing and WES & $\begin{array}{l}\text { - Variety of available target enrichment methods } \\
\text { (e.g., PCR-based, hybridization-based, } \\
\text { computational enrichment by ONT) }\end{array}$ & - Target enrichment may be laborious & [20-22] \\
\hline WGS & $\begin{array}{l}\text { - Molecular diagnosis and pharmacogenetic } \\
\text { profiling in one assay }\end{array}$ & $\begin{array}{l}\text { - Unavailability of PGX-specific analysis tools } \\
\text { for long reads }\end{array}$ & [23] \\
\hline
\end{tabular}

CNV: Copy number variation; NGS: Next-generation sequencing; ONT: Oxford Nanopore Technologies; PacBio: Pacific Biosciences; PGRN: Pharmacogenomics Research Network; PGx: Pharmacogenetics; WES: Whole-exome sequencing; WGS: Whole-genome sequencing.

Indeed, the 2013 European Pharmacogenetics of Anticoagulant Therapy (EU-PACT) [27] and the 2017 Genetic Informatics Trial (GIFT) [28] RCTs as well as a retrospective analysis [29] and a matched control study [30] showed clinical benefits of PGx-guided dosing, whereas in the 2013 Clarification of Optimal Anticoagulation through Genetics (COAG) RCT [31] PGx-guided dosing lead to no significant improvement. These conflicting findings are potentially explained by the fact that in the COAG RCT, $\sim 35 \%$ of the participants were of African descent. Since only the variants $C Y P 2 C 9^{*} 2,{ }^{*} 3$ and $V K O R C 1{ }^{*} 2$ were tested, but not the PM variants $C Y P 2 C 9 * 5,{ }^{*} 6,{ }^{*} 8,{ }^{*} 11$, which are frequent in Africans, carriers of these variants were erroneously assigned the ${ }^{*} 1$ (i.e., wild-type) allele and, thus, were potentially misdosed due to incomplete genetic data.

Moreover, in recent years, several studies have explored the pharmacogenetic landscape on population scale $[7,8,32-$ 35]. These studies showed that rare variants comprise roughly $30-40 \%$ of the variation in pharmacogenes and in some gene families even $>90 \%$ of the PGx variation may be attributed to rare variants. Although there were differences in the results of these studies, they may largely be attributed to the selected genes, the size of studied cohort and whether the study considered all variants or exclusively focused on selected common/functional variants. In general, however, all rare variants in pharmacogenes (i.e., including nonselected ones) can play a role in drug response and, hence, be of clinical relevance. As targeted genotyping cannot detect rare (i.e., non-targeted) variants and the cost per base of NGS decreases, NGS-based pharmacogenetic profiling seems like the logical successor of traditional methods. 


\section{NGS-based pharmacogenetic profiling}

The advent of NGS has transformed the field of genetics by enabling the parallel sequencing of multiple genes using targeted sequencing, the exonic regions of the genome using whole-exome sequencing (WES) or the entire genome using WGS to a large extent [36]. These techniques have been increasingly used not only in the gene diagnostics of rare diseases and in oncology but are also starting to be incorporated into the field of PGx. NGS-based profiling of pharmacogenes enables to expand pharmacogenetic testing from selected variants to entire genes, allowing the detection of rare variants and the identification of novel variant/gene drug associations (Table 1).

Current NGS-based targeted PGx panels include the AmpliSeq Pharmacogenomics Research Panel (thermofisher.com) or PGRNseq [19], enabling deep sequencing of the most frequently affected pharmacogenes. The ongoing eMERGE-PGx project [37] deploys PGRNseq across nine institutions in the USA with the aim to assess pharmacogenetic variation in $\sim 9000$ participants, particularly rare variants, and to generate a large variant repository called SPHINX (emergesphinx.org), partially linked to phenotypic data, allowing the investigation of novel pharmacogenetic variation. Accordingly, this study tackles one of the major barriers of PGx and aims to expand the scope of PGx. Ultimately, both targeted genotyping and targeted sequencing-based pharmacogenetic profiling arguably have the limitation that they need to be performed as a dedicated test for PGx, whereas WGS, rather than WES, offers the possibility of enabling both gene diagnostics and pharmacogenetic profiling in a single test [7].

Indeed, although WES is one of the most widely used and cost-effective NGS technologies allowing the sequencing of the exome, including pharmacogenes, we and others have shown that (i) WES de facto does not enable the sequencing of the entire exome due to missing capture probes and its sensitivity to guanine-cytosine (GC) content [38-40]; and (ii) depending on the applied exome capturing kit, several well-known but non-exonic (i.e., non-captured) pharmacogenetic variants are not interrogated using WES, including CYP2C19*17, CYP $3 A 5^{*} 3$, $U_{G T 1 A 1}{ }^{*} 28 /{ }^{*} 37$ and $\operatorname{VKORC1}{ }^{*} 2$ [7,26]. In the worst case and without appropriate consideration, these limitations may lead to incomplete or false-negative diagnoses and/or to incorrect pharmacogenetic recommendations.

Based on current progress in NGS technologies, it is foreseeable that WGS will over time increasingly replace WES not only in research but also in clinical applications. WGS has the advantage that it overcomes the limitations of WES, in other words, WGS $(60 \times$ PCR-free, PE150) not only allows better (more accurate) sequencing of the exome at appropriate coverage, but also enables sequencing of non-coding regions $[36,38,40]$. Moreover, the continuous and uniform coverage of PCR-free WGS facilitates detection of copy number variations [36,39,40]. As short-read WGS represents the most comprehensive short-read sequencing (SRS) approach, it constitutes a platform for both gene diagnostics and pharmacogenetic profiling in one assay, allowing the identification of both common/known and rare/novel pharmacogenetic variants [7,41]. Indeed, we have recently demonstrated how pharmacogenetic profiling may be integrated into a diagnostic workflow [7]. Briefly, after extracting the genotypes of the current 46 U-PGx variants from the WGS data using dedicated bioinformatics tools, they are interpreted according to the DPWG guidelines, producing a pharmacogenetic report as well as the Medication Safety Card in credit card format $[7,9,42]$. Likewise, the open-source Pharmacogenomics Clinical Annotation Tool represents a similar approach, which accepts WGS (genomic) Variant Call Format ([g]VCF) and Binary Alignment Map (BAM) files as input and generates pharmacogenetic reports according to the CPIC guidelines [43].

Despite being the predominant NGS approach, SRS is subject to inherent limitations due to its read length of typically $\sim 150 \mathrm{bp}$. These short reads may not be unambiguously mapped to the reference genome, particularly in repetitive and/or homologous regions longer than the read length [36]. These regions are collectively termed the 'dead zone' and comprise $\sim 17 \%$ of the reference genome [36], including several pharmacogenes, such as members of the $U G T$ and $C Y P$ gene families. A notable example is $C Y P 2 D 6$, which is challenging to accurately genotype due to its homologous pseudogenes (CYP2D7 and CYP2D8), numerous star alleles and complex structural variation [16].

\section{WGS-based pharmacogenetic profiling of CYP2D6}

Using a subset of the GeT-RM 2019 consensus genotypes for CYP2D6 [44], we have previously benchmarked the bioinformatic tools Astrolabe [45], Aldy [46] and Stargazer [47], which enable CYP2D6 genotyping using SRS data. We and others noticed that Aldy reached the highest accuracy in the WGS-based genotyping of CYP2D6 [7,48]. Recently, a novel approach named Cyrius [49] has been introduced, promising improved accuracy. Here, we expand our previous benchmark with the results of Cyrius, which, however, may be biased in its favor as Cyrius was optimized/trained using the GeT-RM CYP2D6 genotypes [44,49] (Supplementary Table 1). For our expanded benchmarking of WGS-based CYP2D6 variant detection, we assessed the CYP2D6 genotypes and predicted PGx 
(A)

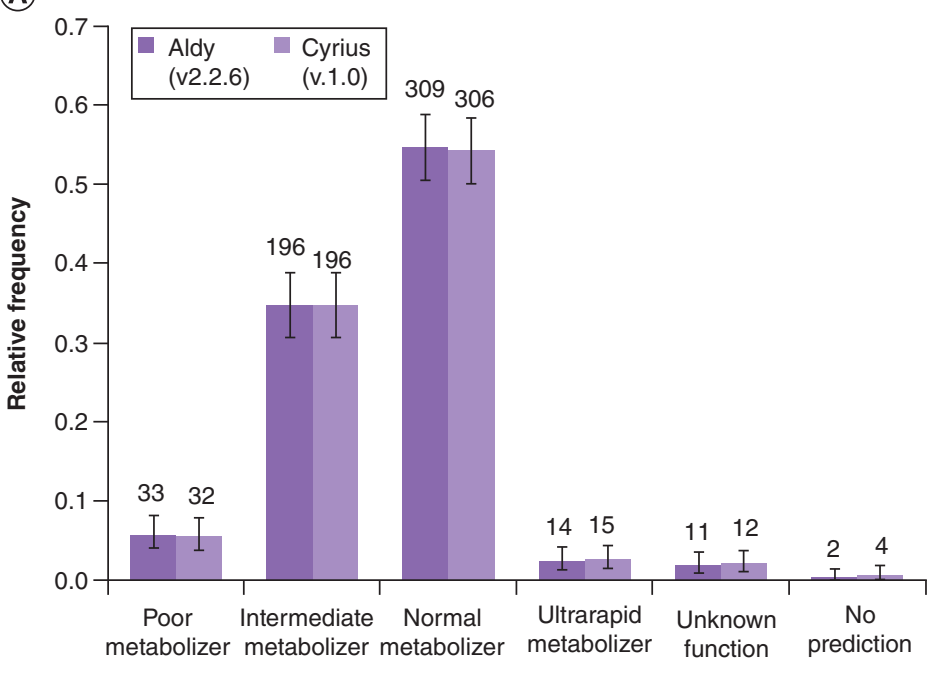

(B)

\begin{tabular}{|ll|}
\hline Concordant & Concordant (BWA) \\
Aldy not called SNV & Complex SV \\
No prediction & CYP2D6*4.013 (*4N) \\
Phasing & \\
\hline
\end{tabular}

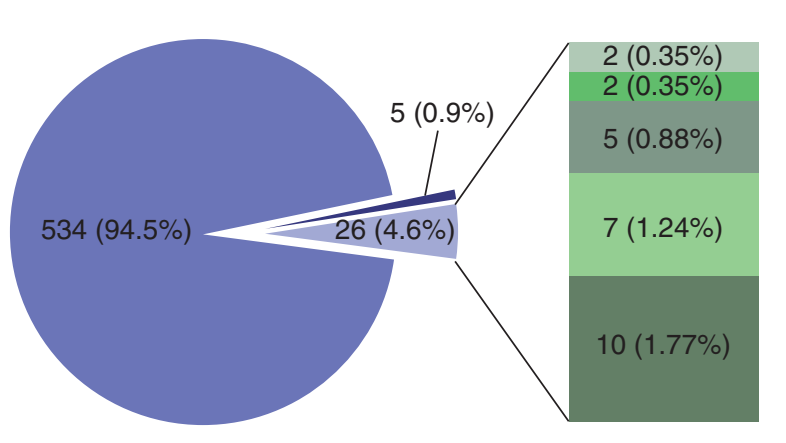

Figure 1. Comparison of Aldy and Cyrius using 565 clinical whole genomes. (A) Relative frequencies of CYP2D6 functional phenotypes predicted by the software tools Aldy and Cyrius using the whole-genome sequencing (60× PCR-free, PE150) data of 565 unrelated samples. Unknown Function: Diplotypes of which the function of at least one allele is unknown. No Prediction: Samples for which the respective tool could not call a CYP2D6 genotype. Error bars indicate $95 \%$ Cls calculated using vassarstats.net/prop1.html (including continuity correction). (B) Number of CYP2D6 genotypes predicted by Aldy and/or Cyrius as well as reason for discordant predictions. Concordant: 534 samples for which Aldy and Cyrius predicted equal/concordant genotypes using Isaac BAM files. Concordant (BWA): 5 of the 31 samples with different/discordant genotypes for which the predictions were identical/concordant using input BAM files aligned with BWA instead of Isaac. No Prediction: Five samples for which neither Cyrius nor Aldy could call a CYP2D6 genotype. The allele CYP2D6*4.013 $(* 4 N)$ is only predicted by Cyrius in seven samples.

SNV: Single nucleotide variant; SV: Structural variant.

phenotypes in 565 primarily Caucasian short-read WGS samples (i) using Aldy [46] compared with Cyrius [49]; (ii) in addition considering rare PGx variants; (iii) exclusively considering the currently implemented U-PGx CYP2D6 alleles.

For (i), WGS (PCR-free, 60× PE150) of 565 unrelated individuals was performed as previously described [40]. Subsequently, FASTQ files were aligned by using Isaac (v.01.15.02.08 or v.04.18.11.09), which is $>5 \times$ faster than the native gold-standard aligner Burrows-Wheeler Aligner (BWA) [50], generating the required input BAM files for Aldy (v2.2.6) and Cyrius (v1.0). Of the 565 analyzed samples, the genotypes and phenotypes were identically predicted by both tools in 534 and 553 samples, which equates to an identical genotype and phenotype detection rate of 94.5 and $97.9 \%$, respectively (Figure 1). We recognize the caveat that the genotypes were mostly not confirmed by an additional method, in other words, we cannot exclude identical genotyping errors of Aldy and Cyrius. In the 534 samples with identical predictions, we identified 24 CYP2D6 haplotypes (Supplementary Table 2) which comprise 76 unique diplotypes (Supplementary Table 3). Of these concordant 534 samples, $\sim 56.0$, $\sim 34.6, \sim 5.1$ and $\sim 2.6 \%$ were classified according to the DPWG guidelines as NM, intermediate metabolizer, PM and ultrarapid metabolizer, respectively (Figure 1). These functional predictions are largely in line with previous estimates for Caucasians $[8,26,51,52]$, but provide additional evidence that complex structural variants (SVs), such as ${ }^{*} 68+{ }^{*} 4$ (relative frequency $6.5 \%$ ), are more common than previously estimated [49].

In addition, for the 31 samples with discordant CYP2D6 genotypes we re-aligned the FASTQ files using BWA v0.7.17 (accelerated with Sentieon [53]), manually evaluated the resulting BAM files using the Integrative Genomics Viewer [54] and re-ran Aldy and Cyrius (Supplementary Table 4). Thereby, five of the 31 discordant samples could be 'rescued' (i.e., the predictions of Aldy and Cyrius were identical/concordant); four cases were due to a novel result of Aldy and one due to a novel result of both, Aldy and Cyrius, indicating the higher sensitivity of Aldy to the alignment tool used for the input BAM file. An important difference between Isaac and BWA is the handling of reads which can be aligned/mapped to multiple positions. By default, Isaac (v.01) aligns such reads to the first possible genomic location [50], whereas BWA aligns such reads randomly [55], which may account for certain 
differences in variant calling and is observable by the number of reads aligned to REP6 and REP7 (Supplementary Figure 1). The most frequent reasons for discordances were because of the allele CYP2D6*4.013 (previously known as ${ }^{*} 4 N$ ) is not covered by Aldy, and therefore, reported as ${ }^{*} 4$ in seven samples as well as due to differences in variant phasing in ten samples (Supplementary Table 4). For example, in one sample Aldy called the diplotype ${ }^{*} 27 /{ }^{*} 41$, while Cyrius called ${ }^{*} 1 /{ }^{*} 32$. Combined, these alleles comprise the same variants but in different phase. Moreover, the tandem rearrangement ${ }^{*} 68+{ }^{*} 4$ was called with a concordance close to $100 \%$, whereas in other rearrangements, including ${ }^{*} 10,{ }^{*} 13$ and ${ }^{*} 36$, the same variants were detected, but on different alleles. Analyzing such discordant results is challenging solely using SRS data and hence orthogonal methods, long-read data or data of family members would be necessary for clarification. However, these discordant genotypes provide important insights, pinpointing which types of variants might be miscalled using such tools.

Both Aldy and Cyrius are easy to use, provide results in less than a minute for WGS input data, do not require high-end hardware, and appear to perform highly accurate and concordant in most cases. Aldy currently supports ten pharmacogenes, while Cyrius is only applicable to CYP2D6. However, Cyrius has the advantages over Aldy of being less sensitive to the alignment software, supporting GRCh38/hg38 in addition to GRCh37/hg19, and being slightly more up-to-date regarding allele designations.

For (ii), in the 534 WGS samples with identical predictions we identified additional CYP2D6 sequence variants using a previously described method [7]. Thereby, we detected 17 coding variants with unknown PGx phenotype (according to pharmvar.org/gene/CYP2D6). One of these variants, NM_000106.5:c.1353G > A p.(Met451Ile), occurred heterozygous only in one sample and was classified as likely deleterious using a PGx-specific variant prediction framework [56 and thus may alter the CYP2D6 activity predicted by Aldy and Cyrius (more details in Supplementary Table 5). Aldy additionally detected possibly functional variants, which are not part of a star allele in the form of appending the suffix '-like' to the identified allele (e.g., ${ }^{*} 1{ }^{*} 2$-like), whereas Cyrius does not report additional detected variants.

For (iii), we limited the analysis of the 534 WGS samples with identical predictions to the 11 CYP2D6 alleles currently tested in the U-PGx project and as recently proposed $\left({ }^{*} 3,{ }^{*} 4,{ }^{*} 5(\mathrm{del}),{ }^{*} 6,{ }^{*} 8,{ }^{*}\right.$, ${ }^{*} 10,{ }^{*} 14,{ }^{*} 17,{ }^{*} 41$, $x N)[9,25]$. In the absence of these variants, the wild-type allele $\left({ }^{*} 1\right)$ was assigned. Accordingly, in the 534 samples we detected 33 unique diplotypes, while 53 of the 76 diplotypes detected by Aldy/Cyrius were incompletely assigned by exclusively considering the current U-PGx alleles (Supplementary Table 3). However, as common but normal functioning alleles including $C Y P 2 D 6{ }^{*} 2,{ }^{*} 33,{ }^{*} 35$ comprise the majority of non-U-PGx alleles, the predicted metabolizer status only differs in a total of eight diplotypes of 12 samples (Supplementary Table 3). Notably, the classification according to the U-PGx system does not enable direct variant phasing. Thus, in the case of duplications, it is not known whether a functional or a nonfunctional allele is duplicated and the system outputs the metabolizer status assuming the most severe phenotype, which represents a major source of discordant phenotype predictions (Supplementary Table 3).

Our data underline that integrating pharmacogenetic profiling into a diagnostic workflow based on WGS is not only feasible and cost efficient, but also largely allows accurate prediction of CYP2D6 genotype and phenotype using appropriate software tools. Moreover, for the example of CYP2D6, our data corroborate the notion that restricting the analysis to the most common and most well-known variants seems to be a useful approach for the majority of individuals. However, similarly as integrating SVs for CYP2D6 phenotype prediction [51], including all known star alleles provides an additional benefit for certain/rare cases. Furthermore, the incorporation of rare non-star-allele variants into pharmacogenetic profiling is at least partly achievable. While loss-of-function (LOF) variants in pharmacogenes, such as nonsense, frameshift or splicing mutations leading to haploinsufficiency (i.e., inactive alleles), may be rather easily interpreted using standard NGS interpretation tools, other variants are more challenging, requiring specialized tools, such as the here applied PGx-specific framework [56], or functional assays. Moreover, variant phasing may be decisive for phenotype prediction but, without additional evidence, it is not possible directly from SRS or targeted genotyping data. Although it is difficult to extrapolate from CYP2D6 to other genes, we assume that the results reported here are likely also applicable to other pharmacogenes to some extent.

Pharmacogenetic profiling using long-read sequencing

Although SRS is powerful, it comes with inherent limitations. In contrast, long-read sequencing (LRS; read lengths $>1$ kilobases) for example, by Pacific Biosciences (pacb.com; PacBio) and Oxford Nanopore Technologies (nanoporetech.com; ONT) has the potential to overcome these limitations, but has often been dismissed for being 
too expensive and having raw-read error rates of $\sim 10 \%$, compared with $<1 \%$ using SRS [36,57]. However, along with other recent seminal studies $[23,58,59]$, the recently published precisionFDA Truth Challenge V2, which aimed to call sequence variants in difficult-to-map regions, emphasized that LRS alone or in combination with SRS is the superior tool for variant calling, phasing and resolving complex or repetitive genomic regions, including several pharmacogenes [60].

Indeed, in the recent years, further technical advances in the form of the PacBio Sequel II (and upcoming Ile) device and the single-molecule real-time (SMRT) Cell 8M allows increased throughput and improved raw-read accuracy. Specifically, by generating and reading circular template DNA molecules multiple times (circular consensus sequencing), high-fidelity reads with an accuracy of $99.8 \%$ can be generated. This is similarly accurate as SRS by Illumina, but with an average read length of $\sim 15$ kilobases, which is sufficient for reads to span the entire CYP2D6 gene and resolve the $H L A$ locus (with read depth $\sim 30 \times$ ) [16,23,58]. The improved raw-read accuracy enables highly contiguious (N50 >15 Mb) de novo human genome assembly, accelerating the process by 10-100-fold, improves variant phasing, and enables accurate single nucleotide variant, indel and SV detection at a read depth of $\sim 15 \times$ coverage [23]. Thereby, a full human genome can be sequenced by high-fidelity sequencing with as few as $2-3$ SMRT cells $8 \mathrm{M}$, decreasing sequencing costs.

The competitor of PacBio, ONT, provides flexible sequencing at various scales, from the smaller Flongle designed for microbiology, to the GridION or PromethION, enabling WGS and up to population-scale sequencing. Indeed, a recent population-scale study has shown that the sequencing of human genomes at $>10 \times$ read depth of coverage with accurate SV detection is possible using a single PromethION flow cell, which contains $~ 3000$ nanopore channels compared with $\sim 500$ on MinION/GridION flow cells [61,62]. Furthermore, the release of ONTs most recent nanopore (R10.3) promises improved raw-read accuracy [63].

For LRS, currently there is no gold standard comparable with BWA/GATK for alignment and variant calling in SRS. Genome assemblies from LRS may be generated using one of the many available assemblers, including FALCON [64], Canu [65], Flye [66], wtdbg2 [67] and Shasta [59]. Consensus accuracy may be further increased by using polishing tools, such as Nanopolish [68], Racon [69] Medaka [70] or Arrow [71], resulting in assemblies similarly accurate as such generated by SRS, but with substantially longer contiguity [72].

In the field of PGx, both PacBio and ONT have been applied for the sequencing of long-range PCR amplicons of the homology-burdened genes CYP2D6, HLA-A and HLA-B to accurately detect and phase haplotypes [73-75]. Moreover, to reduce sequencing costs and increase sequencing read depth, similarly as in SRS, samples may be multiplexed and LRS may be targeted to pharmacogenes. In addition to long-range PCR, pharmacogenes may also be enriched using CRISPR/Cas9-based methods (e.g., [76]) or by hybridization capture [20]. In addition, two recent approaches enable purely computational targeting of genomic regions using ONT, based on real-time selection of DNA molecules of interest using high-end central processing units (CPUs) [21] or graphics processing units (GPUs) [22].

Taken together, the paradigm of accurate SRS and inaccurate LRS reads seems to become increasingly outdated. Although SRS-based CYP2D6 variant callers, such as Aldy and Cyrius, are currently not compatible with LRS, the field of LRS is rapidly growing, driven by the exploration of novel applications and bioinformatic analysis tools of PacBio and ONT LRS by the scientific community (e.g., $[58,77,78]$ ).

\section{Interpretation of genetic variation into actionable guidelines: the final frontier of PGX}

While in the foreseeable future accurate detection and phasing of all sequence variants in an individual's genome is within reach, we and others expect that variant interpretation will remain a challenge for a considerable time to come [79]. Following the sequencing of the first human genome, enormous progress has been made in the in silico interpretation of sequence variants. However, the most efforts of variant effect prediction have focused on the identification of damaging/deleterious variants, in other words, the distinction from neutral variants. In silico algorithms are either based on physiochemical differences among amino acids (e.g., Grantham score [80]), phylogenetic conservation (e.g., GERP, SiPhy) and/or effect on protein structure (e.g., Polyphen-2, SIFT) or ensemble tools combining various existing tools into a single score (e.g., CADD, DANN, Eigen, REVEL) [57]. These algorithms, however, are not optimized for often evolutionary diverse pharmacogenes. Moreover, given that most of these tools have been developed to identify LOF variants, their accuracy for gain-of-function variants is limited [81]. To address these challenges and to more accurately identify functional variants in pharmacogenes, a PGx-specialized framework has recently been developed by combining and adapting the thresholds of several common prediction algorithms [56]. 
These prediction scores, which we also applied to classify functional variants in CYP2D6 (Supplementary Table 5), are designed to classify single nucleotide variants. However, star alleles often comprised several core variants and, for instance, $\sim 50 \%$ of the current CYP2D6 star alleles have an unknown function [79]. Moreover, using the star allele nomenclature and the activity score system, a considerable amount of variability in CYP2D6-mediated drug metabolism remains unexplained [82]. Two recent studies addressed these challenges, both applied deep learning models to predict CYP2D6 function, incorporating and weighing the effects of multiple variants on the same haplotype and confirmed the results with in vitro data [83,84]. In these studies, the models predict enzyme activity on a continuous scale and propose to omit the star allele system for CYP2D6 [84]. Indeed, with more sequencing data available, detected variants in the polymorphic CYP2D6 gene will continue to grow, potentially giving rise to increasingly complex star alleles $[84,85]$. One could argue that the star allele nomenclature, originally developed for a more manageable number of alleles, could be replaced by systems based on continuous scales, which are perhaps more suitable for the myriad of NGS-detected variants.

\section{Current limitations of PGx}

PGx has long been anticipated to be a major driver of improved drug safety and/or efficacy and thereby cost reductions for healthcare systems [14]. Although the success of PGx arguably does not yet have a transformative impact on therapeutic medicine as predicted in the early 2000s [86], scientific evidence is accumulating, indicating that incorporation of PGx into CDS may improve safety and efficacy as well as reduce costs and hospitalization compared with standard treatment [87]. Despite this accumulating evidence, several barriers remain for PGx-based precision medicine in clinical routine. Recent surveys of healthcare professionals showed that, in general, the acceptance of the premise and benefits of PGx is high, while perceived knowledge, lack of clear guidelines and the costs of pharmacogenetic testing still represent barriers [87-91]. Thus, educational resources, such as PharmGKB or the U-PGx e-learning platform $[25,92]$ are highly warranted.

In spite of largely accepted scientific evidence for PGx, some points of discussion still exist, most of which originate from the fact that, currently, PGx fails to explain $100 \%$ of variability in drug response. This could partly be explained by other factors affecting drug response, such as age, sex, co-medication, disease state, kidney and liver function, which may explain up to $60 \%$ of the interindividual variability in drug response [93]. In addition, our current understanding of PGx is still incomplete. For instance, in addition to rare variants, cis- and trans-regulating variants have been identified in pharmacogenes, adding an additional layer of complexity to genotype to phenotype translation [15].

The final step of the implementation of PGx into CDS is a system that informs healthcare professionals either when a PGx test is indicated (pre-test alert) or when an actionable variant has been detected (post-test alert). Ideally, CDS systems should be electronic, easily accessible and provide intuitive guidelines on drug selection and dosing [87]. Several efforts are underway, including approaches to integrate CDS into electronic health records [37,94] or the platform-independent Medication Safety Code initiative [25,42]. The broad establishment of standardized pharmacogenetic testing will be challenging [4] and stakeholders will need to rely on well-founded research to enable its implementation.

\section{Conclusion}

Since the beginning of its implementation, PGx has made substantial progress. Nevertheless, its current real-life applications are imperfect solutions and the impact, optimal platform and evidence of the benefits of PGx remain points of discussion in the scientific community $[9,86,95,96]$. To date, focusing on the most common variants with functional prediction, targeted genotyping still represents the most widely adapted pharmacogenetic profiling approach. However, harboring the limitation that rare but clinically (highly) relevant variants remain undetected, targeted genotyping will continuously be replaced by NGS-based approaches. As the interpretation of NGS-detected rare variants in pharmacogenes is challenging, we advocate that NGS data may in silico be reduced to a subset of variants with existing clinical guidelines. LOF variants as well as rare and putatively clinically relevant variants may be assessed in a research setting and ideally stored in publicly available database $(s)[7,9,37,97]$. To enable the goal of CDS [87], integration of pharmacogenetic information into electronic health records with appropriate alerts or platform-independent systems will be highly valuable.

In the context of rare diseases, diagnostic NGS is routinely performed for screening of disease-causing mutations, irrespective of a suspected pharmacogenetic predisposition. WGS not only generates the most comprehensive sequencing data for mutation screening, but also provides an added value because the data may be used for 
pharmacogenetic profiling as well. Such an approach represents an efficient two-in-one assay without generating significant additional costs. However, the limitations of SRS, particularly inaccessible genomic regions and variant phasing, also apply to PGx. Consequently, LRS either alone or in combination with SRS will not only continue to find application in both research and clinical testing but also in PGx.

\section{Future perspective}

To avoid a further gap between technical advances and their translation into clinical care, well-designed prospective clinical studies (such as the U-PGx PREPARE trial) will provide further evidence for the efficacy of pre-emptive pharmacogenetic testing as a valuable tool of precision medicine. Further technical advances will lead to the abundance of detected sequence variants in pharmacogenes, including such with unknown function/significance. For phenotyping of pharmacogenes, artificial intelligence-based solutions may be coupled and evaluated with high-throughput in vitro functional assays, based on patient-derived, inverse PCR- or CRISPR/Cas9-generated libraries, which has already been demonstrated for CYP2D6 on a smaller scale $[83,84,98]$. Finally, information on PGx in clinical trials is currently limited to oncology [3]. As anticipated in the early 2000s, PGx information may be leveraged for stratification of clinical trials and potentially also for the rescue of dismissed drugs due to previously non-significant results [14].

In addition, the shift of NGS and PGx toward point-of-care testing can be expected, or even toward portable sequencing, for example, using ONT's smartphone-connectable SmidgION [99]. We envision that in the coming years, LRS will be sufficiently mature to usher in the era of long-read genomics and that millions of patients worldwide will have access to high quality sequencing data, furthering the implementation of precision medicine.

Supplementary data

To view the supplementary data that accompany this paper please visit the journal website at: www.futuremedicine.com/doi/sup pl/10.2217/pgs-2020-0155

\section{Author contributions}

SM Caspar, J Meienberg and G Matyas contributed toward conceptualization. SM Caspar, T Schneider, P Stoll and J Meienberg contributed toward data curation. G Matyas contributed toward funding acquisition. SM Caspar, T Schneider, P Stoll, J Meienberg and G Matyas contributed toward methodology. SM Caspar, T Schneider and P Stoll contributed toward software. G Matyas contributed toward supervision. SM Caspar, J Meienberg and G Matyas contributed toward validation. SM Caspar and T Schneider contributed toward visualization. Original draft preparation contributed by SM Caspar. Review and editing of manuscript contributed by SM Caspar, T Schneider, P Stoll, J Meienberg and G Matyas. All authors have read and agreed to the published version of the manuscript.

\section{Acknowledgments}

The authors thank Dr Stephan Fees from bio.logis (bio.logis Genomic Healthcare GmbH, FFM, Germany) for discussions and help with the Genetic Information Management System (GIMS).

\section{Financial \& competing interests disclosure}

This research was funded by the Fondation pour la Recherche et le Traitement Médical (FRTM), Gebauer Stiftung, Lotteriefonds ZH and G + B Schwyzer Stiftung. The authors have no other relevant affiliations or financial involvement with any organization or entity with a financial interest in or financial conflict with the subject matter or materials discussed in the manuscript apart from those disclosed.

No writing assistance was utilized in the production of this manuscript.

Ethical conduct of research

For investigations involving genetic data of human subjects, informed consent has been obtained from the participants involved. 
Executive summary

\section{Current state of pharmacogenetics}

- Pharmacogenetics (PGx) represents a major driver of precision medicine, with the promise of enabling medical treatments tailored to an individual's pharmacogenetic predisposition. Thereby, treatment outcomes may be improved and costs reduced.

- Pharmacogenetic profiling is currently neither the standard in clinical care nor in the stratification of clinical trials.

Approaches for pharmacogenetic profiling: navigating through a labyrinth of possibilities

- A plethora of methods for the genotyping of pharmacogenes exist, but are largely unstandardized. Most providers only interrogate a subset of common/well known variants for which international guidelines exist.

- Rare variants account for $\sim 30-40 \%$ of interindividual variation in pharmacogenes and thereby likely contribute to the variation of individual drug response; however, the functional effects of these variants remain challenging to interpret.

- Next-generation sequencing, such as targeted sequencing of pharmacogenes enables a more comprehensive approach to identify common and rare variants.

Whole-genome sequencing as a platform for pharmacogenetic profiling

- Whole-exome sequencing has the drawback that non-coding but relevant variants, including CYP2C19*17 and CYP3A5*3, are potentially not detected as well as structural variants may be missed.

- We have recently demonstrated that whole-genome sequencing (WGS) may be used for gene diagnostics and PGx in a single assay. Comparing two dedicated bioinformatics tools, in this perspective, we show that short-read sequencing data are largely sufficient for accurate genotyping of the polymorphic CYP2D6 gene.

The future of genetics belongs to long-read sequencing

- As caveats of short-read sequencing include variant phasing and genomic regions with mappability $<1$, long-read sequencing (LRS) provides an alternative which may overcome these challenges.

- Further technical developments of PacBio and Oxford Nanopore Technologies render the paradigm of 'inaccurate LRS' increasingly outdated.

Conclusion \& future perspective

- Although several barriers still exist, PGx has made substantial progress and the results of large-scale studies, such as the ubiquitous pharmacogenomics PREPARE trial, will further promote the field.

- We advocate that if WGS is performed for gene diagnostics, pharmacogenetic profiling may be performed using the existing WGS data without generating significant additional costs.

- We envision that within the next years, LRS will emerge as the predominant sequencing technology and will enable more comprehensive gene diagnostics and PGx.

- Interpretation of the sequence variants will likely remain challenging. Artificial intelligence- or high-throughput in vitro assays will be valuable to overcome this final barrier.

\section{References}

Papers of special note have been highlighted as: • of interest; $\bullet \bullet$ of considerable interest

1. Vogel F. Moderne probleme der humangenetik. In: Ergebnisse der Inneren Medizin und Kinderheilkunde. Heilmeyer L, Schoen R, de Rudder B (Eds). Springer, Berlin, Heidelberg (1959).

2. Relling MV, Klein TE. CPIC: Clinical Pharmacogenetics Implementation Consortium of the Pharmacogenomics Research Network. Clin. Pharmacol. Ther. 89(3), 464-467 (2011).

3. Patel JN. Cancer pharmacogenomics, challenges in implementation, and patient-focused perspectives. Pharmgenomics Pers. Med. 9, 65-77 (2016).

4. Bousman CA, Zierhut H, Müller DJ. Navigating the labyrinth of pharmacogenetic testing: a guide to test selection. Clin. Pharmacol. Ther. 106(2), 309-312 (2019).

5. Ingelman-Sundberg M, Daly AK, Oscarson M, Nebert DW. Human cytochrome P450 (CYP) genes: recommendations for the nomenclature of alleles. Pharmacogenetics 10(1), 91-93 (2000).

6. Bouvy JC, De Bruin ML, Koopmanschap MA. Epidemiology of adverse drug reactions in Europe: a review of recent observational studies. Drug Saf. 38(5), 437-453 (2015).

7. Caspar SM, Schneider T, Meienberg J, Matyas G. Added value of clinical sequencing: WGS-based profiling of pharmacogenes. Int. J. Mol. Sci. 21(7), 2308 (2020).

-. Describes the pipeline which integrates pharmacogenetic profiling into a diagnostic workflow.

8. McInnes G, Lavertu A, Sangkuhl K, Klein TE, Whirl-Carrillo M, Altman RB. Pharmacogenetics at scale: an analysis of the UK Biobank. Clin Pharmacol Ther. doi:10.1002/cpt.2122 (2021) (Epub ahead of print).

9. Van der Wouden CH, van Rhenen MH, Jama WOM et al. Development of the Pgx-passport: a panel of actionable germline genetic variants for pre-emptive pharmacogenetic testing. Clin. Pharmacol. Ther. 106(4), 866-873 (2019). 
10. Mahgoub A, Dring LG, Idle JR, Lancaster R, Smith RL. Polymorphic hydroxylation of debrisoquine in man. Lancet 310(8038), 584-586 (1977).

11. Eichelbaum M, Spannbrucker N, Steincke B, Dengler HJ. Defective N-oxidation of sparteine in man: a new pharmacogenetic defect. Eur. J. Clin. Pharmacol. 16(3), 183-187 (1979).

12. Heim M, Meyer UA. Genotyping of poor metabolisers of debrisoquine by allele-specific PCR amplification. Lancet 336(8714), 529-532 (1990).

13. Wang DG. Large-scale identification, mapping, and genotyping of single-nucleotide polymorphisms in the human genome. Science 280(5366), 1077-1082 (1998).

14. Chicurel ME, Dalma-Weiszhausz DD. Microarrays in pharmacogenomics - advances and future promise. Pharmacogenomics 3(5), 589-601 (2002).

15. Yang Y, Botton MR, Scott ER, Scott SA. Sequencing the CYP2D6 gene: from variant allele discovery to clinical pharmacogenetic testing. Pharmacogenomics 18(7), 673-685 (2017).

16. Nofziger C, Turner AJ, Sangkuhl K et al. PharmVar GeneFocus: CYP2D6. Clin. Pharmacol. Ther. 107(1), 154-170 (2020).

17. Ramírez B, Niño-Orrego MJ, Cárdenas D et al. Copy number variation profiling in pharmacogenetics CYP-450 and GST genes in Colombian population. BMC Med. Genomics 12(1), 110 (2019).

18. Cui J, Wang L, Tan Z, Zhou H, Zhan X, Yin J. Mass spectrometry-based personalized drug therapy. Mass Spec. Rev. 39(5-6), 523-552 (2020).

19. Gordon AS, Fulton RS, Qin X, Mardis ER, Nickerson DA, Scherer S. PGRNseq: a targeted capture sequencing panel for pharmacogenetic research and implementation. Pharmacogenet. Genomics 26(4), 161-168 (2016).

20. Giolai M, Paajanen P, Verweij W, Witek K, Jones JDG, Clark MD. Comparative analysis of targeted long read sequencing approaches for characterization of a plant's immune receptor repertoire. BMC Genomics 18(1), 564 (2017).

21. Kovaka S, Fan Y, Ni B, Timp W, Schatz MC. Targeted nanopore sequencing by real-time mapping of raw electrical signal with UNCALLED. Nat Biotechnol doi:10.1038/s41587-020-0731-9 (2020) (Epub ahead of print).

22. Payne A, Holmes N, Clarke T, Munro R, Debebe B, Loose M. Nanopore adaptive sequencing for mixed samples, whole exome capture and targeted panels. bioRxiv doi:10.1101/2020.02.03.926956 (2020) (Epub ahead of print).

23. Wenger AM, Peluso P, Rowell WJ et al. Accurate circular consensus long-read sequencing improves variant detection and assembly of a human genome. Nat. Biotechnol. 37(10), 1155-1162 (2019).

-• Describes the Pacific Biosciences CCS sequencing method (high-fidelity reads), revealing improved read accuracy, genome assembly and variant calling in a human genome.

24. van der Wouden CH, Bank PCD, Özokcu K, Swen JJ, Guchelaar H-J. Pharmacist-initiated pre-emptive pharmacogenetic panel testing with clinical decision support in primary care: record of PGx results and real-world impact. Genes 10(6), 416 (2019).

25. van der Wouden $\mathrm{CH}$, Cambon-Thomsen A, Cecchin E et al. Implementing pharmacogenomics in Europe: Design and Implementation Strategy of the Ubiquitous Pharmacogenomics Consortium. Clin. Pharmacol. Ther. 101(3), 341-358 (2017).

-. Describes the setup of the ubiquitous pharmacogenomics PREPARE trial, which aims to assess the benefit of pre-emptive pharmacogenetic profiling using targeted genotyping across seven European countries.

26. Reisberg S, Krebs $\mathrm{K}$, Lepamets $\mathrm{M}$ et al. Translating genotype data of 44,000 biobank participants into clinical pharmacogenetic recommendations: challenges and solutions. Genet. Med. 21(6), 1345-1354 (2019).

27. Pirmohamed M, Burnside G, Eriksson N et al. A randomized trial of genotype-guided dosing of warfarin. N. Engl. J. Med. 369(24), 2294-2303 (2013).

28. Gage BF, Bass AR, Lin $\mathrm{H}$ et al. Effect of genotype-guided warfarin dosing on clinical events and anticoagulation control among patients undergoing hip or knee arthroplasty: the GIFT randomized clinical trial. JAMA 318(12), 1115 (2017).

29. Zhang J, Wu T, Chen W, Fu J, Xia X, Chen L. Effect of gene-based warfarin dosing on anticoagulation control and clinical events in a real-world setting. Front. Pharmacol. 10, 1527 (2020).

30. Jorgensen AL, Prince C, Fitzgerald G et al. Implementation of genotype-guided dosing of warfarin with point-of-care genetic testing in three UK clinics: a matched cohort study. BMC Med. 17(1), 76 (2019).

31. Kimmel SE, French B, Kasner SE et al. A pharmacogenetic versus a clinical algorithm for warfarin dosing. N. Engl. J. Med. 369(24), 2283-2293 (2013).

32. Fujikura K, Ingelman-Sundberg M, Lauschke VM. Genetic variation in the human cytochrome P450 supergene family. Pharmacogenet. Genomics 25(12), 584-594 (2015).

33. Kozyra M, Ingelman-Sundberg M, Lauschke VM. Rare genetic variants in cellular transporters, metabolic enzymes, and nuclear receptors can be important determinants of interindividual differences in drug response. Genet. Med. 19(1), 20-29 (2017).

34. Ingelman-Sundberg M, Mkrtchian S, Zhou Y, Lauschke VM. Integrating rare genetic variants into pharmacogenetic drug response predictions. Hum. Genomics 12(1), 26 (2018). 
35. Pandi M-T, Williams MS, van der Spek P, Koromina M, Patrinos GP. Exome-wide analysis of the DiscovEHR cohort reveals novel candidate pharmacogenomic variants for clinical pharmacogenomics. Genes 11(5), 561 (2020).

36. Caspar SM, Dubacher N, Kopps AM, Meienberg J, Henggeler C, Matyas G. Clinical sequencing: from raw data to diagnosis with lifetime value. Clin. Genet. 93(3), 508-519 (2018).

37. Rasmussen-Torvik LJ, Stallings SC, Gordon AS et al. Design and anticipated outcomes of the eMERGE-PGx project: a multicenter pilot for preemptive pharmacogenomics in electronic health record systems. Clin. Pharmacol. Ther. 96(4), 482-489 (2014).

38. Belkadi A, Bolze A, Itan Y et al. Whole-genome sequencing is more powerful than whole-exome sequencing for detecting exome variants. Proc. Natl Acad. Sci. USA 112(17), 5473-5478 (2015).

39. Meienberg J, Zerjavic K, Keller I et al. New insights into the performance of human whole-exome capture platforms. Nucleic Acids Res. 43(11), e76-e76 (2015).

40. Meienberg J, Bruggmann R, Oexle K, Matyas G. Clinical sequencing: is WGS the better WES? Hum. Genet. 135(3), 359-362 (2016).

41. Cohn I, Paton TA, Marshall CR et al. Genome sequencing as a platform for pharmacogenetic genotyping: a pediatric cohort study. $n p j$ Genomic Med. 2(1), 19 (2017).

42. Blagec K, Koopmann R, Crommentuijn - van Rhenen $\mathrm{M}$ et al. Implementing pharmacogenomics decision support across seven European countries: the ubiquitous pharmacogenomics (U-PGx) project. J. Am. Med. Inform. Assoc. 25(7), 893-898 (2018).

43. Sangkuhl K, Whirl-Carrillo M, Whaley RM et al. Pharmacogenomics clinical annotation tool (Pharm CAT). Clin. Pharmacol. Ther. 107(1), 203-210 (2020).

44. Gaedigk A, Turner A, Everts RE et al. Characterization of reference materials for genetic testing of CYP2D6 alleles. J. Mol. Diagn. 21(6), 1034-1052 (2019).

45. Twist GP, Gaedigk A, Miller NA et al. Constellation: a tool for rapid, automated phenotype assignment of a highly polymorphic pharmacogene, CYP2D6, from whole-genome sequences. NPJ Genomic Med. 1(1), 15007 (2016).

46. Numanagić I, Malikić S, Ford $\mathrm{M}$ et al. Allelic decomposition and exact genotyping of highly polymorphic and structurally variant genes. Nat. Commun. 9(1), 828 (2018).

- Presents their SRS-based CYP2D6 variant calling tool called Aldy.

47. Lee S, Wheeler MM, Thummel KE, Nickerson DA. Calling star alleles with stargazer in 28 pharmacogenes with whole genome sequences. Clin. Pharmacol. Ther. 106(6), 1328-1337 (2019).

48. Twesigomwe D, Wright GEB, Drögemöller BI, da Rocha J, Lombard Z, Hazelhurst S. A systematic comparison of pharmacogene star allele calling bioinformatics algorithms: a focus on CYP2D6 genotyping. NPJ Genomic Med. 5(1), 30 (2020).

49. Chen X, Shen F, Gonzaludo N et al. Cyrius: accurate CYP2D6 genotyping using whole genome sequencing data. Pharmacogenomics J doi:10.1038/s41397-020-00205-5 (2021) (Epub ahead of print).

- Presents their SRS-based CYP2D6 variant calling tool called Cyrius.

50. Raczy C, Petrovski R, Saunders CT et al. Isaac: ultra-fast whole-genome secondary analysis on Illumina sequencing platforms. Bioinformatics 29(16), 2041-2043 (2013).

51. Dalton R, Lee S, Claw KG et al. Interrogation of CYP 2D6 structural variant alleles improves the correlation between CYP 2D6 genotype and CYP 2D6-mediated metabolic activity. Clin. Transl. Sci. 13(1), 147-156 (2020).

52. Petrović J, Pešić V, Lauschke VM. Frequencies of clinically important CYP2C19 and CYP2D6 alleles are graded across Europe. Eur. J. Hum. Genet. 28(1), 88-94 (2020).

53. Kendig KI, Baheti S, Bockol MA et al. Sentieon DNASeq variant calling workflow demonstrates strong computational performance and accuracy. Front. Genet. 10, 736 (2019).

54. Robinson JT, Thorvaldsdóttir H, Winckler W et al. Integrative genomics viewer. Nat. Biotechnol. 29(1), 24-26 (2011).

55. Li H, Durbin R. Fast and accurate short read alignment with Burrows-Wheeler transform. Bioinformatics 25(14), 1754-1760 (2009).

56. Zhou Y, Mkrtchian S, Kumondai M, Hiratsuka M, Lauschke VM. An optimized prediction framework to assess the functional impact of pharmacogenetic variants. Pharmacogenomics J. 19(2), 115-126 (2019).

57. Russell LE, Schwarz UI. Variant discovery using next-generation sequencing and its future role in pharmacogenetics. Pharmacogenomics 21(7), 471-486 (2020).

58. Garg S, Fungtammasan A, Carroll A et al. Accurate chromosome-scale haplotype-resolved assembly of human genomes. bioRxiv doi:10.1101/810341 (2019) (Epub ahead of print).

59. Shafin K, Pesout T, Lorig-Roach R et al. Nanopore sequencing and the Shasta toolkit enable efficient de novo assembly of eleven human genomes. Nat. Biotechnol. 38(9), 1044-1053 (2020).

60. PrecisionFDA. Truth challenge V2: calling variants from short and long reads in difficult-to-map regions. (2020). https://precision.fda.gov/challenges/10/view

- In this challenge, several groups submitted their variant call results of downloaded raw data, which were compared with a truth set and ranked according to several metrics. 
61. Beyter D, Ingimundardottir H, Eggertsson HP et al. Long read sequencing of 1,817 Icelanders provides insight into the role of structural variants in human disease. bioRxiv doi:10.1101/848366 (2019) (Epub ahead of print).

62. Oxford Nanopore Technologies. PromethION Brochure. (2019).

https://nanoporetech.com/sites/def ault/files/s3/literature/PromethION-Brochure-14Mar2019.pdf

63. Oxford Nanopore Technologies. The newest nanopore for high accuracy nanopore sequencing. (2020).

https://nanoporetech.com/about-us/news/r103-newest-nanopore-high-accuracy-nanopore-sequencing-now-available-store

64. Chin C-S, Peluso P, Sedlazeck FJ et al. Phased diploid genome assembly with single-molecule real-time sequencing. Nat. Methods 13(12), 1050-1054 (2016).

65. Koren S, Walenz BP, Berlin K, Miller JR, Bergman NH, Phillippy AM. Canu: scalable and accurate long-read assembly via adaptive $k$-mer weighting and repeat separation. Genome Res. 27(5), $722-736$ (2017).

66. Kolmogorov M, Yuan J, Lin Y, Pevzner PA. Assembly of long, error-prone reads using repeat graphs. Nat. Biotechnol. 37(5), 540-546 (2019).

67. Ruan J, Li H. Fast and accurate long-read assembly with wtdbg2. Nat. Methods 17(2), 155-158 (2020).

68. Simpson JT, Workman RE, Zuzarte PC, David M, Dursi LJ, Timp W. Detecting DNA cytosine methylation using nanopore sequencing. Nat. Methods 14(4), 407-410 (2017).

69. Vaser R, Sović I, Nagarajan N, Sikić M. Fast and accurate de novo genome assembly from long uncorrected reads. Genome Res. 27(5), 737-746 (2017).

70. Nicholls SM, Quick JC, Tang S, Loman NJ. Ultra-deep, long-read nanopore sequencing of mock microbial community standards. GigaScience 8(5), giz043 (2019).

71. Amarasinghe SL, Su S, Dong X, Zappia L, Ritchie ME, Gouil Q. Opportunities and challenges in long-read sequencing data analysis. Genome Biol. 21(1), 30 (2020).

72. Logsdon GA, Vollger MR, Eichler EE. Long-read human genome sequencing and its applications. Nat. Rev. Genet. 21(10), 597-614 (2020).

73. Ammar R, Paton TA, Torti D, Shlien A, Bader GD. Long read nanopore sequencing for detection of HLA and CYP2D6 variants and haplotypes. F1000Res 4, 17 (2015).

74. Buermans HPJ, Vossen RHAM, Anvar SY et al. Flexible and scalable full-length CYP2D6 long amplicon PacBio sequencing. Hum. Mutat. 38(3), 310-316 (2017).

75. Liau Y, Maggo S, Miller AL, Pearson JF, Kennedy MA, Cree SL. Nanopore sequencing of the pharmacogene CYP2D6 allows simultaneous haplotyping and detection of duplications. Pharmacogenomics 20(14), 1033-1047 (2019).

76. Gilpatrick T, Lee I, Graham JE et al. Targeted nanopore sequencing with Cas9-guided adapter ligation. Nat. Biotechnol. 38(4), 433-438 (2020).

77. Hon T, Mars K, Young G et al. Highly accurate long-read HiFi sequencing data for five complex genomes. Sci Data. 7(1), 399 (2020).

78. Shumate A, Zimin AV, Sherman RM et al. Assembly and annotation of an Ashkenazi human reference genome. Genome Biol. 21 (1), 129 (2020).

79. Nofziger C, Paulmichl M. Accurately genotyping CYP2D6: not for the faint of heart. Pharmacogenomics 19(13), 999-1002 (2018).

80. Grantham R. Amino acid difference formula to help explain protein evolution. Science 185(4154), 862-864 (1974).

81. Zhou Y, Fujikura K, Mkrtchian S, Lauschke VM. Computational methods for the pharmacogenetic interpretation of next generation sequencing data. Front. Pharmacol. 9, 1437 (2018).

82. Gaedigk A, Dinh J, Jeong H, Prasad B, Leeder J. Ten years' experience with the CYP2D6 activity score: a perspective on future investigations to improve clinical predictions for precision therapeutics. JPM 8(2), 15 (2018).

83. McInnes G, Dalton R, Sangkuhl K et al. Transfer learning enables prediction of CYP2D6 haplotype function. PLoS Comput Biol. 16(11), e1008399 (2020).

84. van der Lee M, Allard WG, Vossen RHAM et al. A unifying model to predict variable drug response for personalised medicine. bioRxiv doi:10.1101/2020.03.02.967554 (2020) (Epub ahead of print).

85. Caudle KE, Sangkuhl K, Whirl-Carrillo M et al. Standardizing CYP 2D6 genotype to phenotype translation: consensus recommendations from the Clinical Pharmacogenetics Implementation Consortium and Dutch Pharmacogenetics Working Group. Clin. Transl. Sci. 13(1), 116-124 (2020).

86. Joyner MJ, Paneth N. Promises, promises, and precision medicine. J. Clin. Invest. 129(3), 946-948 (2019).

87. Krebs K, Milani L. Translating pharmacogenomics into clinical decisions: do not let the perfect be the enemy of the good. Hum. Genomics 13(1), 39 (2019).

88. Stanek EJ, Sanders CL, Taber KAJ et al. Adoption of pharmacogenomic testing by US physicians: results of a nationwide survey. Clin. Pharmacol. Ther. 91(3), 450-458 (2012). 
89. de Denus S, Letarte N, Hurlimann T et al. An evaluation of pharmacists' expectations towards pharmacogenomics. Pharmacogenomics 14(2), 165-175 (2013).

90. Bank PC, Swen JJ, Guchelaar H-J. A nationwide survey of pharmacists' perception of pharmacogenetics in the context of a clinical decision support system containing pharmacogenetics dosing recommendations. Pharmacogenomics 18(3), 215-225 (2017).

91. Just KS, Steffens M, Swen JJ, Patrinos GP, Guchelaar H-J, Stingl JC. Medical education in pharmacogenomics - results from a survey on pharmacogenetic knowledge in healthcare professionals within the European pharmacogenomics clinical implementation project ubiquitous pharmacogenomics (U-PGx). Eur. J. Clin. Pharmacol. 73(10), 1247-1252 (2017).

92. Just KS, Turner RM, Dolžan V et al. Educating the next generation of pharmacogenomics experts: global educational needs and concepts. Clin. Pharmacol. Ther. 106(2), 313-316 (2019).

93. Shah RR. Genotype-guided warfarin therapy: still of only questionable value two decades on. J. Clin. Pharm. Ther. 45(3), 547-560 (2020).

94. Hinderer M, Boeker M, Wagner SA et al. Integrating clinical decision support systems for pharmacogenomic testing into clinical routine - a scoping review of designs of user-system interactions in recent system development. BMC Med. Inform. Decis. Mak. 17(1), 81 (2017).

95. Damkier P. Obviously nine believers: actionable germline genetic variants for pre-emptive pharmacogenetic testing. Basic Clin. Pharmacol. Toxicol. 126(1), 5-6 (2020).

96. Gaedigk A. Pharmacogenetics: chasing perfection. Clin. Pharmacol. Ther. 106(2), 265-270 (2019).

97. Lauschke VM, Ingelman-Sundberg M. Requirements for comprehensive pharmacogenetic genotyping platforms. Pharmacogenomics 17(8), 917-924 (2016).

98. Chiasson MA, Rollins NJ, Stephany JJ et al. Multiplexed measurement of variant abundance and activity reveals VKOR topology, active site and human variant impact. eLife 9 , e58026 (2020).

99. Oxford Nanopore Technologies. Smidgion (2020). https://nanoporetech.com/products/smidgion 